Annales Geophysicae (2001) 19: 275-287 (C) European Geophysical Society 2001

\title{
Magnetic field fluctuations across the Earth's bow shock
}

\author{
A. Czaykowska ${ }^{1}$, T. M. Bauer ${ }^{1}$, R. A. Treumann ${ }^{1,2,3}$, and W. Baumjohann ${ }^{1,4}$ \\ ${ }^{1}$ Max-Planck-Institut für extraterrestrische Physik, Garching, Germany \\ ${ }^{2}$ Centre for Interdisciplinary Plasma Science, Garching, Germany \\ ${ }^{3}$ International Space Science Institute, Bern, Switzerland \\ ${ }^{4}$ Institut für Weltraumforschung der Österreichischen Akademie der Wissenschaften, Graz, Austria
}

Received: 25 September 2000 - Revised: 10 January 2001 - Accepted: 24 January 2001

\begin{abstract}
We present a statistical analysis of 132 dayside (LT 0700-1700) bow shock crossings of the AMPTE/IRM spacecraft. We perform a superposed epoch analysis of low frequency, magnetic power spectra some minutes upstream and downstream of the bow shock. The events are devicded into categories depending on the angle $\theta_{B n}$ between bow shock normal and interplanetary magnetic field, and on plasma- $\beta$. In the foreshock upstream of the quasiparallel bow shock, the power of the magnetic fluctuations is roughly 1 order of magnitude larger $(\delta B \sim 4 \mathrm{nT}$ for frequencies $0.01-0.04 \mathrm{~Hz}$ ) than upstream of the quasi-perpendicular shock. There is no significant difference in the magnetic power spectra upstream and downstream of the quasi-parallel bow shock; only at the shock itself, is the magnetic power enhanced by a factor of 4 . This enhancement may be due to either an amplification of convecting upstream waves or to wave generation at the shock interface. On the contrary, downstream of the quasi-perpendicular shock, the magnetic wave activity is considerably higher than upstream. Downstream of the quasi-perpendicular low- $\beta$ bow shock, we find a dominance of the left-hand polarized component at frequencies just below the ion-cyclotron frequency, with amplitudes of about $3 \mathrm{nT}$. These waves are identified as ioncyclotron waves, which grow in a low- $\beta$ regime due to the proton temperature anisotropy. We find a strong correlation of this anisotropy with the intensity of the left-hand polarized component. Downstream of some nearly perpendicular $\left(\theta_{B n} \approx 90^{\circ}\right)$ high- $\beta$ crossings, mirror waves are identified. However, there are also cases where the conditions for mirror modes are met downstream of the nearly perpendicular shock, but no mirror waves are observed.
\end{abstract}

Key words. Interplanetary physics (plasma waves and turbulence) - Magnetospheric physics (magnetosheath; plasma

Correspondence to: R. A. Treumann (tre@mpe.mpg.de) waves and instabilities)

\section{Introduction}

Since the beginning of the space age, the Earth's bow shock is of particular interest because it serves as a unique laboratory for the study of shock waves in collisionless plasmas. Most of our understanding of structure, dynamics, and dissipation processes of such collisionless shocks has come from in situ spacecraft measurements crossing the bow shock. A collection of observational and theoretical work on the bow shock is contained in Stone and Tsurutani (1985) and Tsurutani and Stone (1985). Here we are mainly interested in waves related to the region just behind the bow shock. We discuss the wave mode spectra like the mirror mode, the ioncyclotron mode in relation to upstream wave spectra. The measurements used have been obtained by the AMPTE/IRM spacecraft during passages of the Earth's bow shock and magnetosheath. Most of the observations are from the region of the bow shock around noon and morning, and from a radial distance interval $R>11 \mathrm{R}_{\mathrm{E}}$. Since IRM had an apogee of $\sim 18 R_{E}$, sometimes the spacecraft was, for a long time, in the bow shock and the adjacent magnetosheath region, covering primarily a weakly depressed magnetosheath comparable to those reported by Lacombe et al. (1995), who observed mirror-like waves and other kinds of waves, both there and in the bow shock itself. Similar observations reaching even deeper into the magnetosheath have been presented by $\mathrm{Hu}-$ bert et al. (1998). These observations, like ours, refer to a "normal" or relatively quiet magnetosheath. Complementary observations in a highly compressed magnetosheath, compressed even to cross the path of AMPTE/CCE, which, at quiet conditions, was found deep in the magnetosphere, have been discussed by Anderson et al. (1993, 1994). 
Structure, dynamics, and dissipation processes of the bow shock vary considerably, depending on the angle $\theta_{B n}$ between the upstream magnetic field and the shock normal, on the plasma- $\beta$, i.e., the ratio of plasma to magnetic pressure in the upstream region, and on the Mach numbers $M_{A}$ or $M_{m s}$, i.e., the ratios of the solar wind velocity along the shock normal to the upstream Alfvén or magnetosonic speed. For quasi-perpendicular shocks with $\theta_{B n}>45^{\circ}$, the main transition from the solar wind to the magnetosheath is accomplished at a sharp ramp. In contrast, quasi-parallel shocks with $\theta_{B n}<45^{\circ}$ consist of large-amplitude pulsations extending into the foreshock region. For larger Mach numbers, this pulsating structure continuously re-forms by virtue of collisions between convecting upstream waves and the shock (Burgess, 1989), or due to an instability at the interface between solar wind and heated downstream plasma (Winske et al., 1990).

Two-fluid theories of shocks have indicated the presence of a critical magnetosonic Mach number, $M^{*}$, above which ion reflection is required to provide the necessary dissipation (e.g. Kennel et al., 1985). However, it has been demonstrated by observations (Greenstadt and Mellott, 1987; Sckopke et al., 1990) that ion reflection occurs also below $M^{*}$ and that the distinction between subcritical $\left(M_{m s}<M^{*}\right)$ and supercritical $\left(M_{m s}>M^{*}\right)$ shocks is not sharp. Whereas the ramp of quasi-perpendicular shocks at high Mach numbers is preceded by a foot and followed by an overshoot, these features are less prominent at low Mach numbers (Mellott and Livesey, 1987). While quasi-parallel shocks are steady at low Alfvén Mach numbers, $M_{A} \leq 2.3$, they become unsteady for higher Mach numbers due to their continuous reformation (Krauss-Varban and Omidi, 1991; Winske et al., 1990; Omidi, 1995).

An important role in the dissipation process is played by ions reflected at the bow shock (Sckopke et al., 1983; Thomsen et al., 1985). At quasi-parallel shocks, they can escape from the shock into the foreshock region and drive ion beam instabilities. These instabilities may excite large-amplitude waves observed in the foreshock region, e.g. by Le and Russell (1992) and Blanco-Cano and Schwartz (1995). At quasiperpendicular shocks, the reflected ions gyrate back to the shock and enter the downstream region, where their presence causes a high perpendicular temperature anisotropy (Sckopke et al., 1983). This anisotropy leads to the generation of ioncyclotron and mirror waves (e.g. Price et al., 1986; Gary et al., 1993). These waves have been observed in the Earth's magnetosheath, e.g. by Sckopke et al. (1990) and Anderson et al. (1993, 1994). Closer to the magnetopause, the mechanism of field line draping generates anisotropic ion distributions and leads to the formation of the plasma depletion layer. Waves in this environment have also been described by Anderson et al. (1993, 1994). Large-amplitude mirror waves have been observed in planetary magnetosheaths, e.g. by Bavassano-Cattaneo et al. (1998) in Saturn's magnetosheath, where the ion temperature anisotropies are due to both shock heating and field line draping.

In the present study, we investigate the average be-

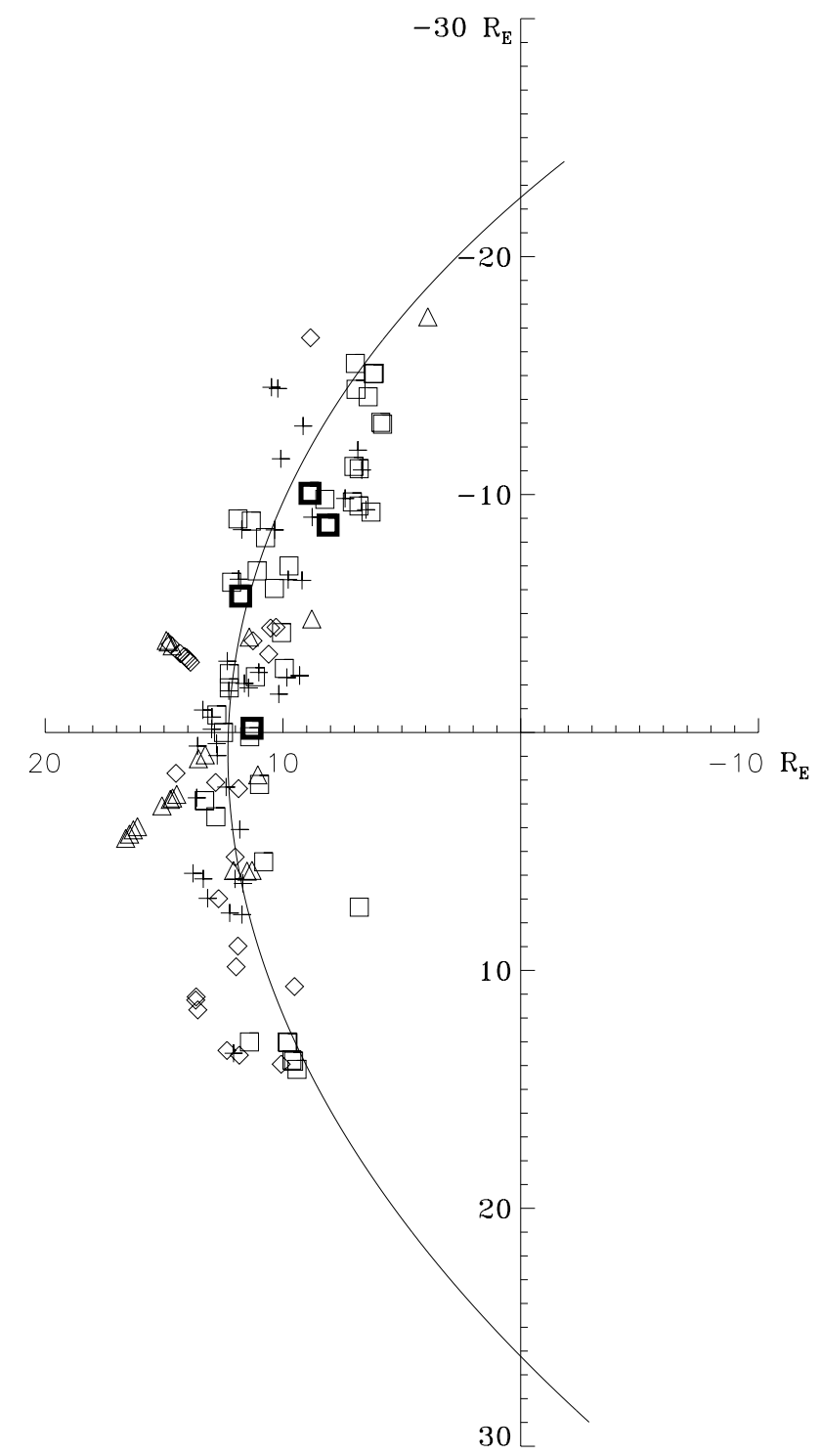

Fig. 1. Positions of the 132 AMPTE IRM bow shock crossings rotated into the ecliptic plane: quasi-parallel are + signs; quasiperpendicular low- $\beta(\beta<0.5)$ are triangles,; quasi-perpendicular medium- $\beta(0.5<\beta<1.0)$ are diamonds; quasi-perpendicular high- $\beta(\beta>1.0)$ are squares; bold squares are events, where mirror modes have clearly been identified. The hyperbola is the best-fit hyperbola through the crossings with multiple crossings weighted as one event.

haviour of the low frequency magnetic wave power as measured by AMPTE/IRM during 132 bow shock crossings. We show that these waves behave differently across quasiperpendicular and quasi-parallel shocks. Section 2 provides a short description of the available data. It is followed by Sect. 3 which compares quasi-perpendicular and quasiparallel shock crossings. In Sect. 4 , low and high- $\beta$ bow shock crossings are compared for the quasi-perpendicular shock, and it is outlined why a classification by $\beta$ is preferred over the classification by Mach number. 


\section{Data description and method of analysis}

The present analysis uses data from the AMPTE/IRM satellite. From the periods when the apogee of AMPTE/IRM was on the Earth's dayside (August - December 1984 and August 1985 - January 1986), we have selected all crossings of the satellite through the Earth's bow shock in the local time interval $0700-1700$, whenever there was a reasonable amount of data measured on both sides of the bow shock, i.e. at least 2 min upstream and 4 min downstream. Altogether, this gives 132 events, with some events belonging to multiple crossings due to the fast movement of the bow shock relative to the slow moving satellite. Due to the satellite's orbital parameters, all crossings occurred at low latitudes, i.e. in the interval $\pm 30^{\circ}$ from the ecliptic plane. A geometrical overview of all crossings relative to the best average position of the bow shock is given in Fig. 1. The symbols in this figure refer to parallel and perpendicular bow shocks and will otherwise become clear later in the text. The four highlighted squares refer to our best cases of mirror mode observations. The crossings are rarely evenly distributed in real time. In determining the average bow shock position, we have chosen to count multiple crossings within a short time sequence as one single crossing. This leads to larger scatter in the data, but does not put too much weight on fast time fluctuations in the shock position. We analyze the data from the triaxial fluxgate magnetometer described by Lühr et al. (1985), which gives the magnetic field vector at a rate of 32 samples per second. In addition, we use the plasma moments calculated from the three-dimensional particle distribution functions measured once every spacecraft revolution $(\sim 4.3 \mathrm{~s})$ by the plasma instrument (Paschmann et al., 1985).

In order to obtain the average behaviour of plasma and magnetic field parameters at the bow shock, one would ideally need average spatial profiles of these parameters. However, with just one satellite and in a region with strong plasma flows and strong motions of the region itself, it is not unambiguously possible to translate the time profiles into spatial profiles. Therefore, we perform a superposed epoch analysis by averaging time profiles centered on the bow shock crossing time and consider the result as an approximation for the average spatial behaviour. The time series are aligned on the keytime with the upstream always preceding, i.e. for outbound crossings, the time sequence had to be reversed. One may expect that the recently launched multi-spacecraft mission CLUSTER will contribute to a better clarification of the related questions.

\section{Comparison of quasi-perpendicular and quasi-parallel bow shock crossings}

We divided the 132 events into 92 quasi-perpendicular $\left(\theta_{B n}>45^{\circ}\right)$ and 40 quasi-parallel $\left(\theta_{B n}<45^{\circ}\right)$ cases and compared the average behaviour of plasma and magnetic field parameters and low frequency magnetic fluctuations of the two groups.
Actually, for the quasi-parallel bow shock crossings, $\theta_{B n}$ varies substantially with time in the dynamic foreshock region. For these events, the angle $\theta_{B n}$ had to be averaged over a time interval of about $20 \mathrm{~s}$ further upstream to identify them with quasi-parallel shock crossings. The high level of fluctuations in the region upstream of the quasi-parallel bow shock is well known (e.g. Hoppe et al., 1981; Greenstadt et al., 1995). The keytime, i.e. the bow shock crossing time, is identified with the steepest drop in the proton velocity. This drop is well defined for the quasi-perpendicular cases and, of course, corresponds to shock ramp crossing. Due to the large-amplitude pulsations in the foreshock, the keytime cannot as easily be determined in the quasi-parallel cases. We therefore used, as an additional criterion for quasiparallel events, the condition that no solar wind-like plasma is allowed to be visible in the downstream region. As noted in Sect. 1, quasi-parallel shocks consist of large-amplitude pulsations associated with a sequence of partial transitions from solar wind-like to magnetosheath-like plasma and vice versa. Thus, our definition of the keytime implies that the keytime of quasi-parallel shocks corresponds to the downstream end of this pulsating transition region.

We use data from 2 min upstream to 4 min downstream for the analysis of the plasma and magnetic field parameters, and data from 3 min upstream to 9 min downstream for the low frequency fluctuations, although such long time profiles are not available for all events.

In order to analyze the low frequency magnetic fluctuations, we perform a spectral analysis of the magnetic field using a cosine-bell filter (e.g. Bauer et al., 1995). The Fourier transform is taken over a time interval of $4 \mathrm{~min}$. Figures 2 and 3 show the resulting power spectra of the compressive and the right- and left-hand polarized modes, respectively. In each graph, the center time $(-3,-1,1,3,5,7)$ of the transformed time interval is given in minutes, relative to crossing time. A cross marks the proton cyclotron frequency $f_{c p}=$ $e B / 2 \pi m_{p}$ with $m_{p}$ the proton mass. In Fig. 2, the solar wind (SW) spectrum 3 min upstream of the quasi-perpendicular bow shock shows a structureless decrease to higher frequencies following a power law $S \sim f^{-1.3}$. The compressive mode lies below the transverse modes that represent Alfvén waves, which are frequently observed in the interplanetary medium. They are believed to have their origin in the vicinity of the Sun (Belcher and Davis, 1971). The spectrum upstream of the quasi-parallel bow shock has much higher power than the spectrum upstream of the quasi-perpendicular bow

shock. It also has a different structure: for lower frequencies, it shows a flatter decrease $\left(S \sim f^{-0.5}\right)$, while for higher frequencies, it decreases more steeply $\left(S \sim f^{-2.0}\right)$. The kink in the spectrum lies below the proton cyclotron frequency.

The next power spectra $(-1$, SW/BS $)$ contain magnetic field data from the upstream region and the bow shock itself. At the quasi-perpendicular bow shock, the intensity of the compressive mode at low frequencies is one order of magnitude higher compared to that of the transverse modes. It follows a power law of $S \sim f^{-2.1}$. This law simply repre- 


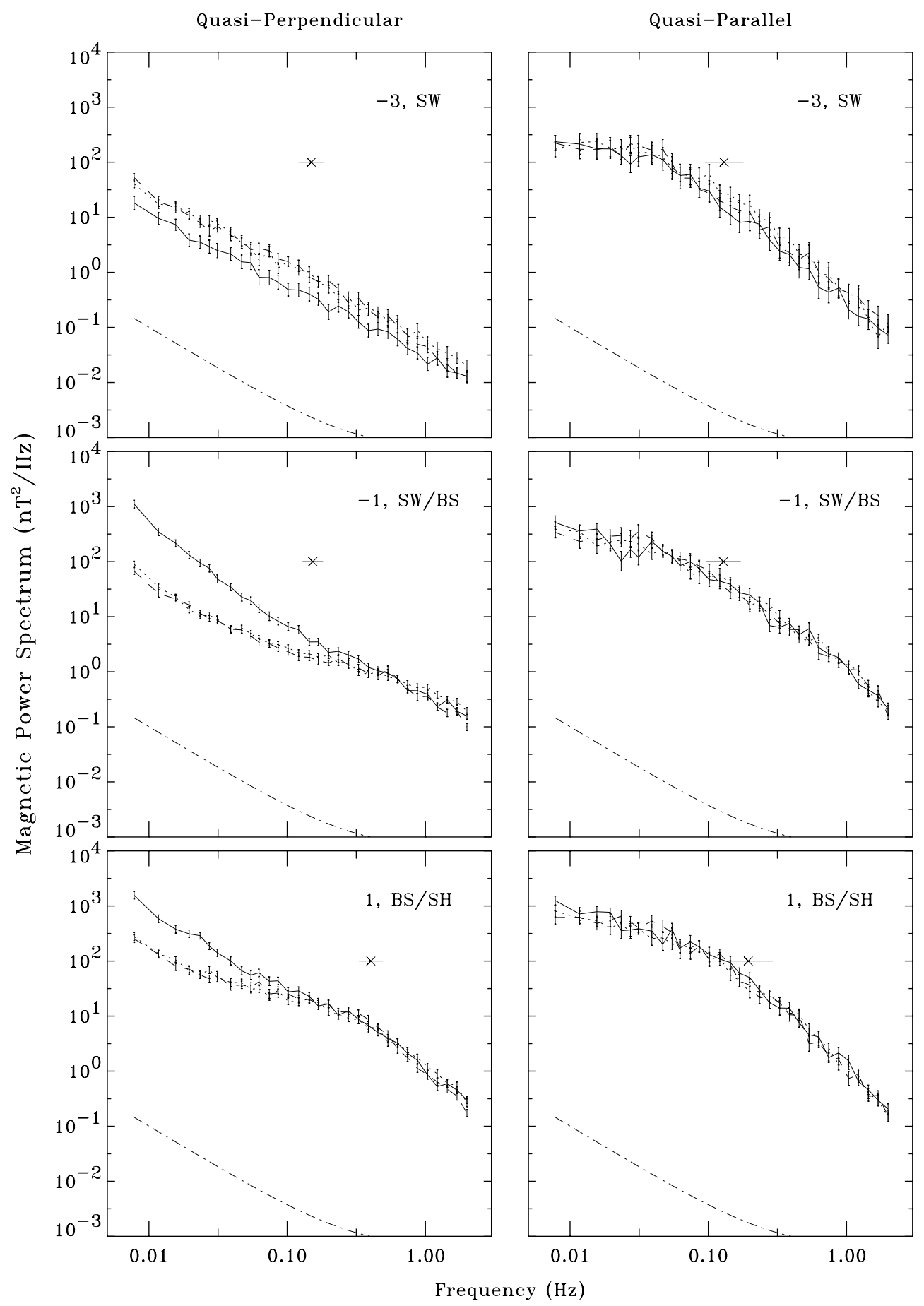

Fig. 2. Superposed-epoch analysis of magnetic spectra of 92 quasi-perpendicular (left) and 40 quasi-parallel bow shock crossings (right) from 3 min upstream to 1 min downstream. Solid line is a compressive component; dashed line is a left hand polarized component; dotted line is a right hand polarized component. The cross with the horizontal error bar marks the proton cyclotron frequency. The acronyms SW, BS, SH mean solar wind, bow shock, and magnetosheath, respectively. Dash-dot line at the bottom of each figure is the instrumental noise level.

sents the spectrum of the jump of the magnetic field across the shock when filtered with the cosine bell function (see Bauer et al., 1995). The spectra of the transverse modes are slightly more intense than had been observed 2 min earlier. In addition, the spectral decrease with frequency turns out to be no longer constant. At the quasi-parallel bow shock, the increase of the magnetic field is practically invisible. This behaviour is not surprising since the magnetic field increases only gradually. Both the level and structure of the spectrum are similar to those calculated at the time 2 min earlier. 


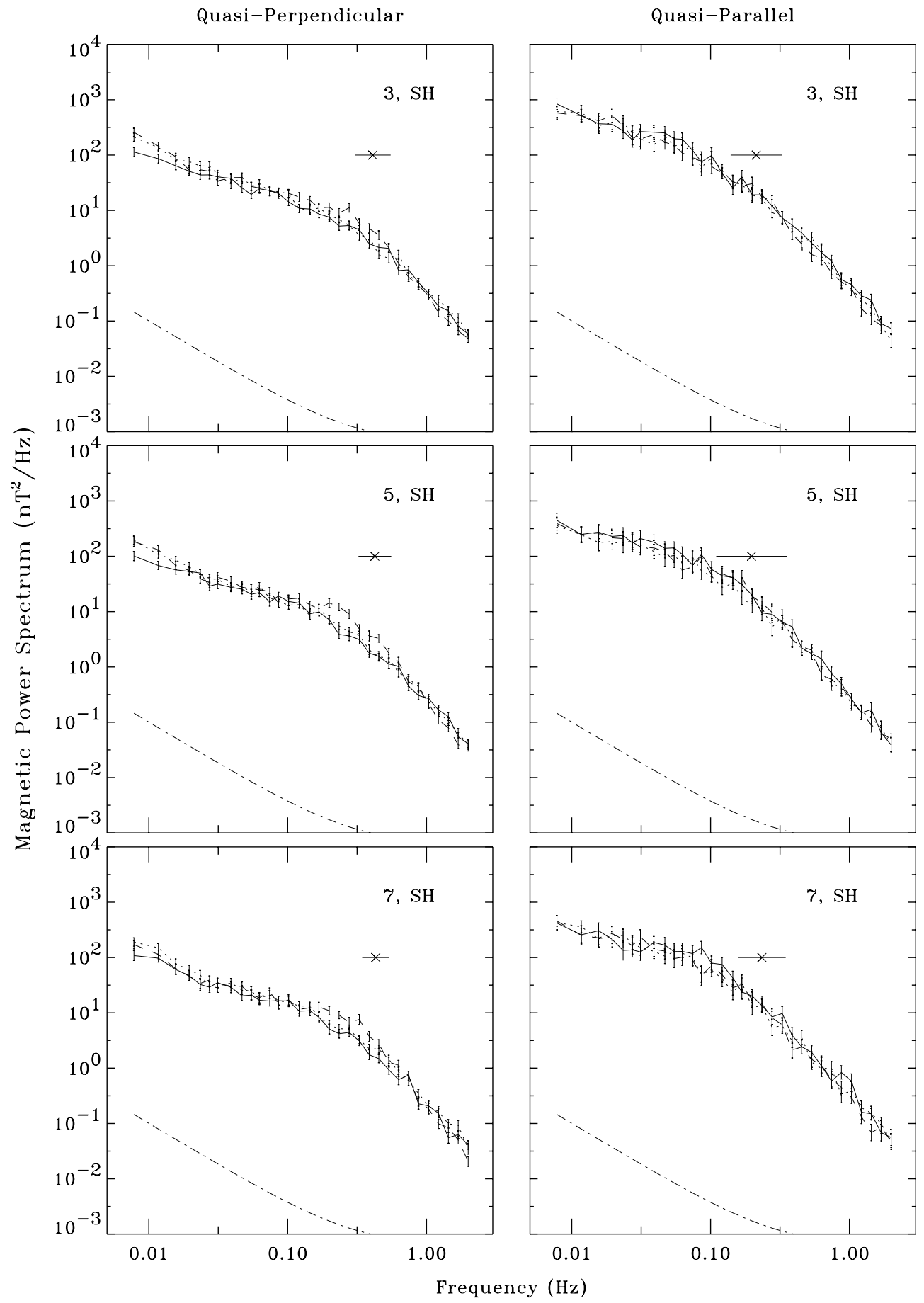

Fig. 3. Superposed-epoch analysis of magnetic spectra of 92 quasi-perpendicular (left) and 40 quasi-parallel bow shock crossings (right) from $3 \mathrm{~min}$ to $7 \mathrm{~min}$ downstream. Same format as Fig. 2.

The spectra (1, BS/SH) contain magnetic field data from again the bow shock itself, and from the magnetosheath, just downstream of the bow shock. At the quasi-perpendicular bow shock, the compressive mode behaves similar to the spectrum 2 min earlier, whereas the spectral power of the transverse modes is higher than 2 min earlier. Just below the proton cyclotron frequency, first indications of plateau formation are visible. At the quasi-parallel bow shock, wave activity is significantly enhanced by a factor of 4 , compared to the upstream spectra. Again, all three modes behave similar. The proton cyclotron frequencies increase according to the magnetic field increase by a factor of 3 at the quasiperpendicular bow shock, and by a factor of 2 at the quasiparallel bow shock.

Figure 3 shows that for both categories of shock crossings, the spectra do not change much in the interval 2 to $8 \mathrm{~min}$ 
downstream of the bow shock. Below $f_{c p}$, the compressive and the right-hand polarized modes downstream of the quasiperpendicular bow shock follow a power law $S \sim f^{-1.1}$, whereas the spectral energy of the left-hand polarized mode is clearly enhanced in the frequency interval from about $0.1 \mathrm{~Hz}$ to about the proton cyclotron frequency. This fact is investigated more carefully in Sect. 4. Downstream of the quasi-parallel bow shock, the spectral energy is again higher than downstream of the quasi-perpendicular bow shock. For $f<f_{c p}$, it follows the power law $S \sim f^{-0.8}$. Three min after the crossing, the spectral energy is higher than in the two later spectra, but already lower than directly at the bow shock. For both categories, the spectral energy decreases steeply $\left(S \sim f^{-2.6}\right)$ for $f>f_{c p}$.

At the quasi-parallel bow shocks, the wave power observed 3 min upstream of the keytime is much higher than the power of the interplanetary Alfvén waves observed upstream of quasi-perpendicular shocks. This enhanced power reflects upstream waves generated in the foreshock. Observations of upstream waves have been reviewed by Greenstadt et al. (1995) and Russell and Farris (1995). The nonlinear steepening of the shock leads to whistler precursors phase standing in the shock frame. The interaction between ions reflected at the shock and the incoming solar wind drives ion beam instabilities which are the source of the large-amplitude waves observed at periods around $30 \mathrm{~s}$. Finally, there are upstream propagating whistlers with frequencies around $1 \mathrm{~Hz}$ generated right at, or close, to the shock ramp. The most striking feature in the average spectrum of upstream waves observed $3 \mathrm{~min}$ before keytime is the kink at $0.04 \mathrm{~Hz} \approx f_{c p} / 3$. The average power measured in the flat portion $0.01-0.04 \mathrm{~Hz}$ of the spectrum corresponds to a mean square amplitude $\delta B_{1} \approx 4 \mathrm{nT}$ or $\delta B_{1} / B \approx 0.4$. Large-amplitude waves observed in this frequency range (Le and Russell, 1992; Blanco-Cano and Schwartz, 1995) have been interpreted as either upstream propagating magnetosonic waves excited by the right-hand resonant ion beam instability, upstream propagating Alfvén/ion-cyclotron waves excited by the left-hand resonant ion beam instability, or non-resonant downstream propagating magnetosonic waves. Whereas the upstream propagating magnetosonic waves should be left-hand polarized in the shock frame (and also in the spacecraft frame), the other two wave types should be right-hand polarized. This might explain why none of the two circular polarizations dominates in our average spectra. Moreover, the compressional component is comparable to the two transverse components. This shows that the waves propagate at oblique angles to the magnetic field. For oblique propagation, low frequency waves have only a small helicity (Gary, 1986). Thus, they are rather linearly rather than circularly polarized.

The power spectra presented by Le and Russell (1992) exhibit clear peaks at $f \approx f_{c p} / 3$. Looking into the spectra of individual time intervals, we find that sometimes the IRM data exhibit similar spectral peaks. However, most of the individual spectra do not have clear peaks, but are rather flat in the range $0.01-0.04 \mathrm{~Hz}$, like the average spectrum of Fig. 2. The steep decrease of the power above around $f \approx f_{c p} / 3$ is common to our spectra and those reported previously. In fact, the maximum growth rate of the ion beam generated waves is expected for frequencies below the proton cyclotron frequency (e.g. Scholer et al., 1997).

At the keytime at quasi-parallel bow shocks, we observe a clear enhancement in wave power. This enhancement is either due to amplification of upstream waves or to wave generation at the shock interface. Wave generation at the shock due to interface instability has been reported from hybrid simulations of Winske et al. (1990) and Scholer et al. (1997). This instability is driven by the interaction between incoming solar wind ions and the heated downstream plasma at the shock interface. Amplification of upstream waves has been predicted long ago by McKenzie and Westphal (1969), who theoretically analyzed the transmission of MHD waves across fast shocks. According to them, the amplitude of Alfvén waves increases by a factor of $\sim 3$. For compressional waves, the amplification can be even stronger. However, hybrid simulations by Krauss-Varban (1995) show that the transmission of waves across the shock is considerably complicated by mode conversion.

The proton temperature anisotropy downstream of the quasi-perpendicular bow shock (see below) serves as a source of free energy. According to both observations and simulations, this kind of free energy drives two modes of low frequency waves under the plasma conditions in the magnetosheath: the ion-cyclotron wave and the mirror mode (see e.g. Sckopke et al., 1990; Hubert et al., 1989; Lacombe et al., 1992, and Anderson et al., 1994, for observations, Price et al., 1986; and Gary et al., 1993, for simulations, and Schwartz et al., 1996, for a review).

Which of these waves grow under which conditions will be investigated more closely in Sect. 4 , where we divide the crossings of quasi-perpendicular shocks into cases with low and high upstream $\beta$, respectively. For quasi-parallel bow shocks, we were not able to investigate the difference between subcritical and supercritical shocks, since no subcritical quasi-parallel shock was identified in the data set. By trying higher thresholds for the division into low and high Mach numbers, we did not find any qualitative differences. In this context, it should be noted that only one of the cases in our data set has an Alfvén Mach number in the range $M_{A} \leq 2.3$, for which quasi-parallel shocks are steady, according to the hybrid simulations of Krauss-Varban and Omidi (1991). The classification by $\beta$ is superior to a classification by the upstream Mach number, since the critical Mach number, $M^{*}$, above which, ion reflection is required to provide the necessary dissipation, depends strongly on $\beta$ (Edmiston and Kennel, 1984). The ratio $M_{m s} / M^{*}$ for our shock crossings suggests that all subcritical shocks are low- $\beta$, i.e. the classification low- $\beta$ versus high- $\beta$ is more or less identical to the classification subcritical versus supercritical. At large $\beta$ the critical Mach number decreases, such that $M^{*} \rightarrow 1$ for $\beta \gg 1$. As the excitation of mirror and ion-cyclotron waves depends on $\beta$, the results of Sect. 4 should be interpreted as a $\beta$ rather than Mach number effect. 


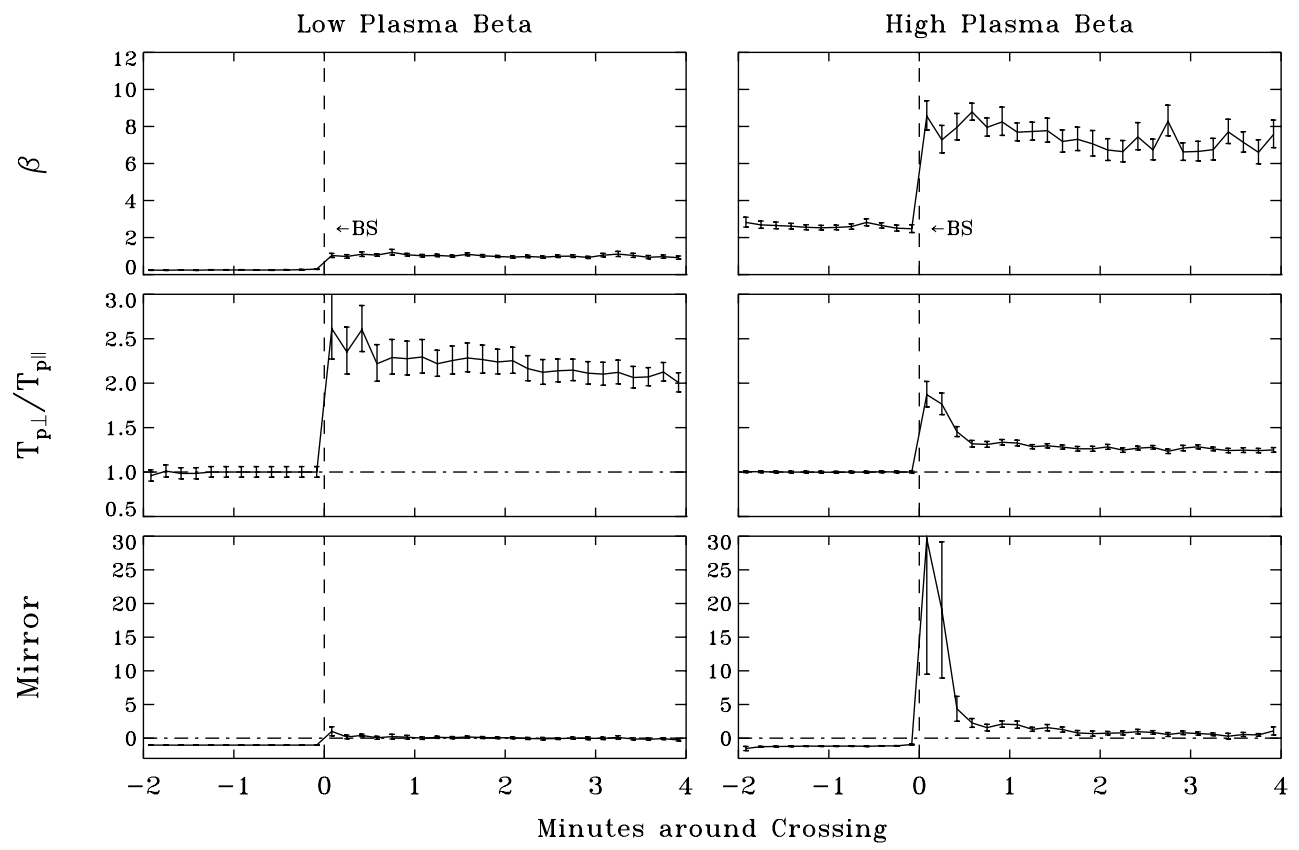

Fig. 4. Superposed-epoch analysis of the plasma parameter $\beta$, the proton temperature anisotropy $T_{p \perp} / T_{p \|}$, and the mirror instability criterion from 2 min upstream to 4 min downstream of the quasi-perpendicular bow shock (BS) on the left for 20 low- $\beta$, on the right of 47 high- $\beta$ crossings.

\section{Comparison of quasi-perpendicular low- $\beta$ and high- $\beta$ bow shock crossings}

In this section, we discuss the properties of the circularly polarized left-hand and right-hand component in the power spectra downstream of the quasi-perpendicular bow shock (Fig. 3). We divide the crossings into classes with low (< $0.5)$ and high $(>1.0)$ upstream $\beta$ and compare these two classes. There are 20 low- $\beta$ and 47 high- $\beta$ cases. The crossings with $0.5 \leq \beta \leq 1.0$ are not included in this analysis in order to emphasize the differences between the low- $\beta$ and high- $\beta$ regimes.

In Fig. 4 we show some interesting differences between the low $-\beta$ and high- $\beta$ categories. Of course the plasma parameter $\beta$ differs essentially. In the upstream region, $\beta$ is derived by setting the proton density to the corrected electron density and the proton temperature to $10^{5} \mathrm{~K}$. This is the long-term average of the proton temperature which is used here since, in the cold solar wind stream, proton distribution functions are not well resolved (Sect. 3). The classification for the low- $\beta$ and high- $\beta$ categories is derived from the estimated plasma- $\beta$ in the upstream region. The first panel of Fig. 4 shows that the same classification could be obtained using the plasma- $\beta$ of the downstream region with the limits shifted to larger values. The most striking differences between the low- $\beta$ and high- $\beta$ bow shock are shown in the next two panels, i.e. the proton temperature anisotropy $T_{p \perp} / T_{p \|}$ and the mirror wave instability criterion. Both parameters are determined only in the downstream region. The instability criterion for almost perpendicular propagation of the mirror mode in an anisotropic cold electron plasma is in its general form (Hasegawa, 1969) given by

$-1+\sum_{j} \beta_{j \perp}\left(\frac{\beta_{j \perp}}{\beta_{j \|}}-1\right)>0$.

The subscript $j$ denotes different ion species. Note that in the cold electron limit, the electrons do not appear in this formula.

Downstream of the quasi-perpendicular low- $\beta$ bow shock, the proton temperature anisotropy is very high, $T_{p \perp} / T_{p \|} \approx$ 2.5 , immediately behind the shock and remains high, $T_{p \perp} /$ $T_{p \|}>2$, throughout the whole magnetosheath interval investigated. Downstream of the quasi-perpendicular high- $\beta$ bow shock, the proton temperature anisotropy is also significant, but lower, compared to the low- $\beta$ bow shock, i.e. $T_{p \perp} / T_{p \|} \approx 1.8$ just behind the bow shock and $T_{p \perp} / T_{p \|} \approx$ 1.3 further downstream. The mirror instability criterion is only marginally satisfied immediately downstream of the quasi-perpendicular low- $\beta$ bow shock and is not satisfied at later times, since the instability criterion depends not only on the particle temperature anisotropy, but also on the absolute value of $\beta$. Downstream of the quasi-perpendicular high- $\beta$ bow shock, the mirror instability criterion is satisfied in the entire interval of 4 min behind the shock. Extremely high values of the left-hand side of Eq. (1) are occasionally observed immediately behind the shock.

Figure 5 shows the magnetic power spectra downstream of the quasi-perpendicular low- $\beta$ (left) and high- $\beta$ (right) bow shock, at 3, 5, and $7 \mathrm{~min}$ after the crossing time. From this figure, it is obvious that the left-hand polarized mode only dominates just behind the quasi-perpendicular bow shock in 


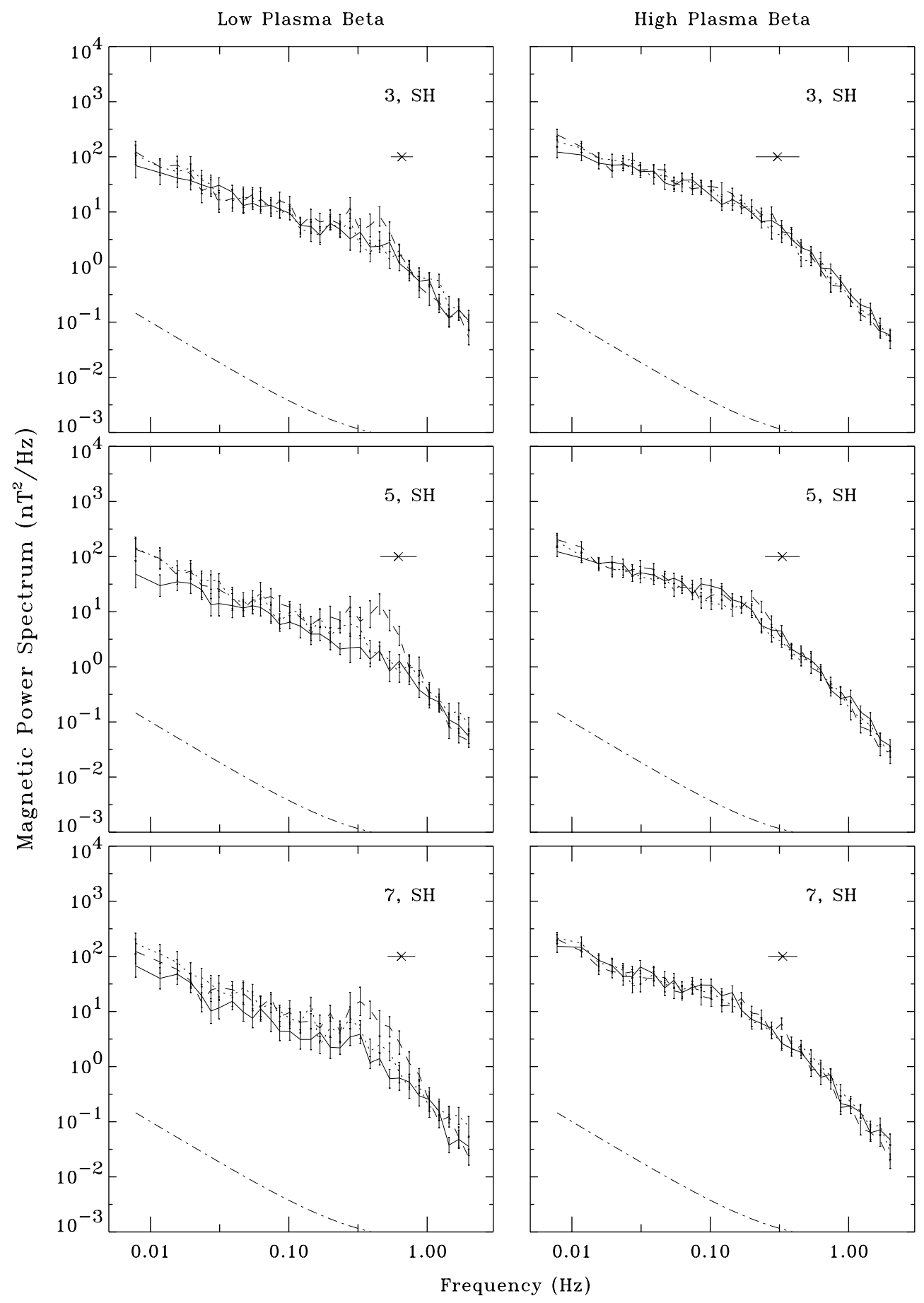

Fig. 5. Superposed-epoch analysis of magnetic power spectra downstream of 20 quasi-perpendicular low- $\beta$ and 47 quasi-perpendicular high- $\beta$ bow shock crossings. Same format as Fig. 3 .

the low- $\beta$ region for about 5 and $7 \mathrm{~min}$ in the frequency interval below the proton cyclotron frequency. Most of the power is in the left-hand polarized mode which is up to one order of magnitude more intense than both the compressive and right-hand polarized components, respectively. The quasiperpendicular high- $\beta$ bow shock downstream spectrum below this frequency range is flatter than downstream of the low- $\beta$ bow shock. In addition, the intensity of the compres- sive mode is at the same level as the transverse modes and occasionally, even more intense, whereas 5 and 7 min downstream of the low- $\beta$ bow shock, the compressive mode is clearly weaker than both transverse modes. Due to the higher magnetic field, the proton cyclotron frequency is a factor of about $\sim 2$ higher downstream of the low- $\beta$ bow shock $(B \approx$ $45 \mathrm{nT})$ than downstream of the high- $\beta$ bow shock $(B \approx 23$ nT). 


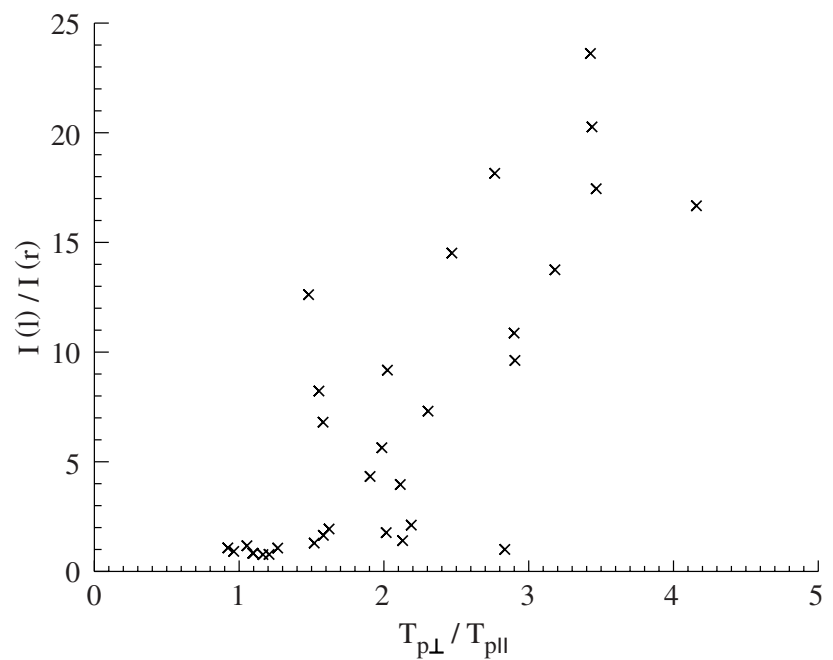

Fig. 6. Ratio of the left-hand polarized to the right-hand polarized component $I(l) / I(r)$ against the proton temperature anisotropy $T_{p \perp} / T_{p \|}$ of 322 -min intervals from 4 to 8 min downstream of the quasi-perpendicular low- $\beta$ bow shock.

In order to obtain a feeling for the typical wave amplitudes, we introduce the root mean square amplitudes $\delta B_{1}$ and $\delta B_{2}$, obtained by integrating the power spectra in the frequency range $0.01-0.04 \mathrm{~Hz}$ and above $0.23 \mathrm{~Hz}$, respectively. The range $0.01-0.04 \mathrm{~Hz}$ corresponds to the flat portion of the spectrum observed 3 min upstream of the keytime of the investigated quasi-parallel bow shocks. These upstream waves have $\delta B_{1} \approx 4 \mathrm{nT}$ or $\delta B_{1} / B \approx 0.4$. In contrast, $\delta B_{1} \approx 0.8 \mathrm{nT}$ for Alfvén waves in the solar wind upstream of the quasi-perpendicular bow shocks, correspond to a ratio $\delta B_{1} / B \approx 0.09$. The former $40 \%$ amplitude indicates very large amplitude waves. Even the latter, indicating 9\% amplitudes, can be considered large amplitude. Such waves behave nonlinearly and have important consequences in the reflection and acceleration of particles as well as in the dissipative processes in the plasma. At the keytime of quasi-parallel bow shocks, we observed a pronounced enhancement of the wave power with fluctuation amplitude $\delta B \approx 8 \mathrm{nT}$ and $\delta B / B \approx 0.5$.

In conclusion, the characteristics of the wave activity downstream of quasi-perpendicular shocks depend on $\beta$. For low $\beta$, the magnetic fluctuations above $0.23 \mathrm{~Hz}$ are dominated by left-hand polarized fluctuations with $\delta B_{2} \approx 3 \mathrm{nT}$ or $\delta B_{2} / B \approx$ 0.08 , which will be interpreted as ion-cyclotron waves. For high $\beta$, typical amplitudes are $\delta B_{2} \approx 1.5 \mathrm{nT}$ or $\delta B_{2} / B \approx$ 0.08 .

Sckopke et al. (1990) have performed a case study of low$\beta$ subcritical bow shock crossings, using the AMPTE/IRM data of 5 September and 2 November 1984. These events are included in our quasi-perpendicular low- $\beta$ data set; there are 8 events from 5 September and 3 events from 2 November 1984. Sckopke et al. (1990) identified the dominating left-hand polarized component with the ion-cyclotron wave, which can be generated by proton temperature anisotropy

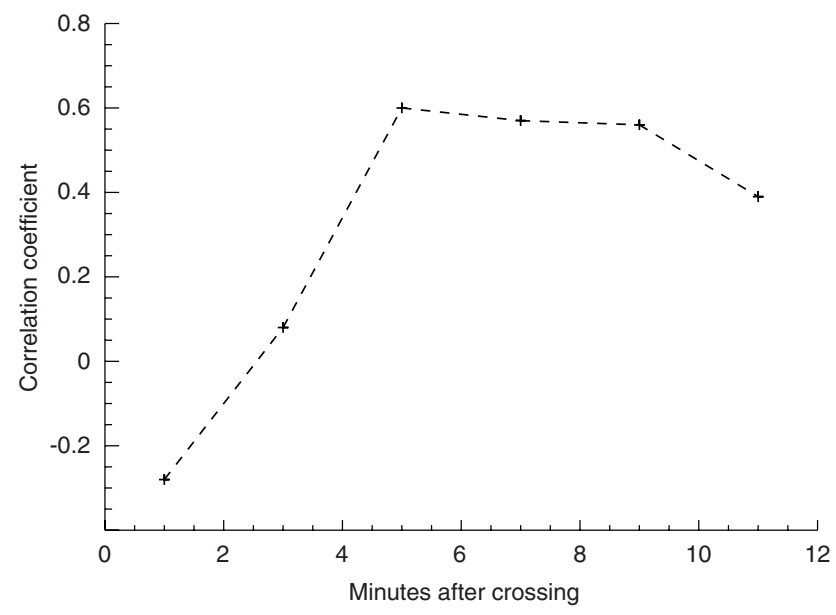

Fig. 7. Temporal evolution of the correlation coefficient of the ratios $I(l) / I(r)$ and $T_{p \perp} / T_{p \|}$ downstream of the quasi-perpendicular low- $\beta$ bow shock.

(e.g., Hasegawa, 1975). The growth rate of the ion-cyclotron wave is positive when the instability criterion is satisfied. This holds for resonance with protons whenever

$\frac{T_{p \perp}}{T_{p \|}}>\frac{f_{c p}}{f_{c p}-f}$.

The AMPTE/IRM plasma instrument did not resolve ion masses; therefore, all ions are assumed to be protons.

Figure 6 shows the ratio of the left-hand polarized to the right-hand polarized component in the frequency band 0.3 $0.8 f_{c p}$ for 32 2-min intervals from 4 to 8 min downstream of the quasi-perpendicular low- $\beta$ bow shock, as a function of the proton temperature anisotropy. There is a clear correlation found between these two ratios with correlation coefficient $\sim 0.8$. This shows that the wave intensity of more than 4 min downstream of the quasi-perpendicular bow shock depends strongly on the local temperature anisotropy.

The temporal evolution of the same correlation coefficients, calculated for 2-min intervals downstream of the quasi-perpendicular low- $\beta$ bow shock, is given in Fig. 7. The value of the correlation coefficient 11 minutes downstream is not reliable since only a limited data set extends so far downstream. Although the temperature anisotropy is highest immediately downstream of the bow shock, the best correlation is found around $5 \mathrm{~min}$ downstream. This shows that the ion-cyclotron waves need a certain time to develop in the moving plasma.

Since downstream of the quasi-perpendicular high- $\beta$ bow shock the mirror instability criterion is satisfied on average, we have looked more carefully for this highly compressive, non-propagating mode. The fact that the compressive mode has a higher power spectral density downstream of the high- $\beta$ than downstream of the low- $\beta$ shock might indicate the existence of mirror modes. We, therefore, perform a superposed epoch analysis for 9 cases, for which the mirror instability criterion is particularly well satisfied, 7 min downstream of the high- $\beta$ bow shock. The resulting spectrum is shown in 


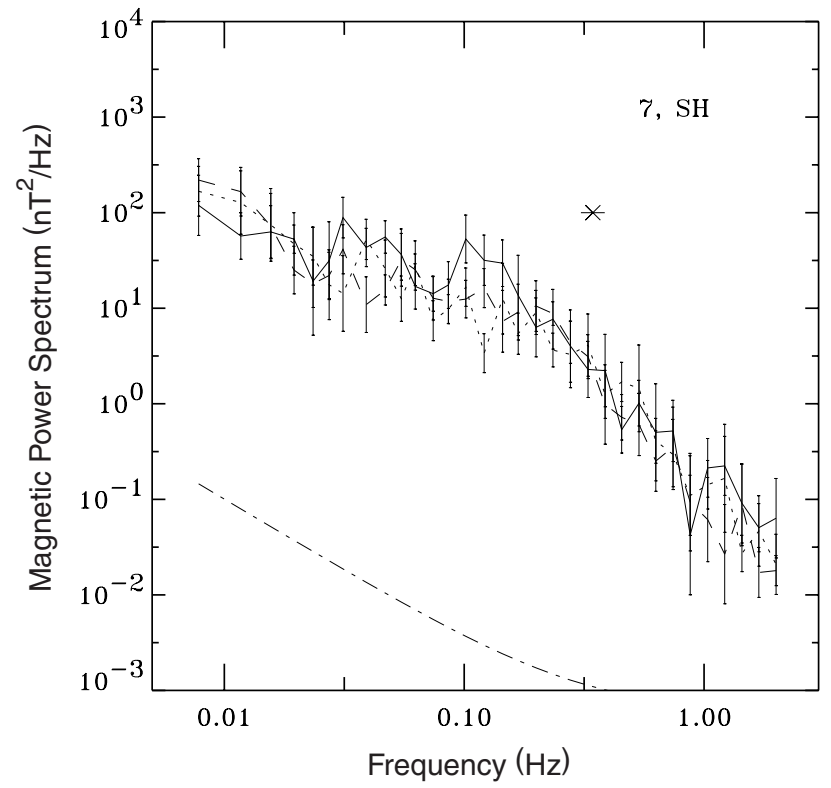

Fig. 8. Superposed-epoch analysis of magnetic spectra 7 min downstream of 9 quasi-perpendicular high- $\beta$ bow shock crossings of which the mirror instability criterion is well satisfied. The dash-dot line at the bottom stands for the instrumental noise level.

Fig. 8. In this spectrum, we find a compressive mode only slightly dominating at frequencies well below the proton cyclotron frequency.

Contrary to the correlation of the intensity of the left-hand polarized ion-cyclotron waves to the proton temperature anisotropy for quasi-perpendicular low- $\beta$ cases, our investigation does not reveal a clear correlation of the intensity of the compressive mode to any parameter for quasi-perpendicular high- $\beta$ cases. The reason for this is probably that the highly compressive mirror mode, which is expected to exist under the observed high- $\beta$ conditions, is a purely growing mode with real frequency existing as pure Doppler-shifted frequencies. Consequently, the waves do not appear in a fixed frequency interval and can be smeared out in the superposition. Therefore, we have looked into the individual spectra of intervals 5,7 , and 9 min downstream of the quasiperpendicular high- $\beta$ bow shock when the mirror criterion is fulfilled.

This is the case in 34 of the quasi-perpendicular high- $\beta$ events $(72 \%)$. Only in 4 cases (12\% of the quasi-perpendicular high- $\beta$ events where the mirror criterion is fulfilled) can mirror waves clearly be identified on the magnetosheath side of the bow shock and are they visible in several consecutive spectra. The best case presented in the case study of Czaykowska et al. (1998) was at radial distance of $12.8 \mathrm{R}_{\mathrm{E}}$ and $\sim 0900$ MLT, corresponding to normal conditions when the sheath was rather weakly compressed. However, even in these cases, ion-cyclotron waves are present though, they do not dominate the spectra. These events and the means of identification of the mirror modes have been described in detail by Czaykowska et al. (1998). The 4 events have, in common, the angle $\theta_{B n}$ greater than $80^{\circ}$. There are also high$\beta_{\perp}$ events with large values of the left-hand side of Eq. (1) where no indication for mirror waves could be recognized. These cases are purely determined by ion-cyclotron waves. For 14 of the high- $\beta$ events $(41 \%)$, where the mirror instability criterion is fulfilled, the compressive component is slightly dominating at several frequencies. Several of these events have an angle $\theta_{B n}<80^{\circ}$. In addition, 2 high- $\beta$ events show ion-cyclotron waves in the consecutive spectra taken 5 , 7 , and 9 min downstream. Thus, we conclude that, in this study, we have practically not found any case of pure mirror modes in the magnetosheath region under investigation, i.e. in the magnetosheath closely adjacent to the bow shock wave. The mirror modes detected here are all accompanied by ion-cyclotron waves and, only in a few cases, energetically dominate the wave spectrum.

It is known (e.g. Price et al., 1986; Lee et al., 1988; Gary et al., 1993) that the instabilities of ion-cyclotron and mirror waves are competing processes. In the magnetosheath plasma, the crucial parameters for this competition are the $\alpha$-particle concentration and plasma- $\beta$. In our data set, we found many events in the high- $\beta$ regime where none of the two wave modes could in anyway be identified, although apparently, the proton temperature anisotropy was high. The reasons for this observation are not known. What concerns the ion-anisotropy driven ion-cyclotron mode, one might argue, is that it is Landau damped by the nearly isotropic thermal background electrons. In the case of the mirror mode, the observations indicate that its mechanism of generation is not yet thoroughly clarified. Inclusion of finite electron temperature has been demonstrated to drastically reduce its growth rate due to Landau damping by field aligned electron heat flux (e.g. Pantellini, 1995; Pantellini and Schwartz, 1998; Pokhotelov et al., 2000a). Moreover, kinetic effects (e.g. Pokhotelov et al., 2000b) may further lower its growth rate and preclude its evolution. Ultimately, nonlinear effects, which even today are poorly understood, may contribute to its suppression. Concerning the latter, it is well known by now that quasi-linear theory (Treumann and Baumjohann, 1997) does not properly describe the non-linear behaviour of the mirror mode. Instead, the zero-parallel energy ions play a decisive role in its saturation (Kivelson and Southwood, 1996), leading to deformation of the ion phase space distribution function. Subsequent secondary instabilities may transfer their energy to other modes until the mirror mode dies out. Its absence in the data signifies that this transfer happens at a rather fast rate. Statistical studies of measurements in the magnetosheath suggest that a relation of the form

$\frac{T_{p \perp}}{T_{p \|}}-1=\frac{S}{\left(\beta_{p \|}\right)^{\alpha}}$

exists between the proton temperature anisotropy, and the ratio $\beta_{p \|}$ of field-aligned proton pressure and magnetic pressure. Analyzing AMPTE/CCE data, Anderson et al. (1994) obtained $S=0.85$ and $\alpha=0.48$, and Fuselier et al. (1994) estimated $S=0.83$ and $\alpha=0.58$. Using AMPTE/IRM data, Phan et al. (1994) found, in the vicinity of the magne- 
topause, $S=0.58$ and $\alpha=0.53$. We performed a similar analysis on our data set of quasi-perpendicular bow shock crossings. For this analysis we computed 2-min averages of all measurements of $T_{p \perp} / T_{p \|}$ and $\beta_{p \|}$ taken between keytime and 8 min downstream of keytime. We find a reasonable fit to Eq. (3) with $S=0.43 \pm 0.03$ and $\alpha=0.58 \pm 0.05$, which is smaller than the values given by Anderson et al. and Fuselier et al., while closer to the results of Phan et al. (1994). The difference may be sought in the different magnetosheath conditions for the Anderson et al. and our cases, respectively. Anderson et al., with CCE measured during highly disturbed conditions of a strongly compressed shockmagnetosphere system, when CCE at about $6-8 R_{E}$, was in the magnetosheath, while our IRM measurements were obtained during "normal" shock-sheath transitions. Thus, one expects that our fluctuation level would be weaker than that of Anderson et al., in agreement with our findings. Phan's et al. result shows that, under such normal conditions, this level increases toward the magnetopause while the exponent $\alpha$ on $\beta_{p}$ remains practically constant. The larger value for Anderson's et al. case agrees with the idea of a higher magnetosheath proton pressure anisotropy under strongly compressed conditions.

A relation of the form of Eq. (3) is regarded as the consequence of the combined action of ion-cyclotron and mirror waves, which grow, due to temperature anisotropy, and reduce this anisotropy by means of pitch angle scattering. The growth of the waves depends on $T_{p \perp} / T_{p \|}$ and $\beta_{p \|}$. It is expected that the anisotropy is reduced until the growth rate, $\gamma_{m}$, of the most unstable wave falls below some threshold. In fact, Anderson et al. (1994) showed that Eq. (3) with their values of $S$ and $\alpha$ corresponds approximately to the threshold $\gamma_{m} / 2 \pi f_{c p}=0.01$. Hence, the validity of Eq. (3) indicates that the magnetosheath plasma reaches a state near marginal stability of the waves driven by the temperature anisotropy. As noted above, we found that data obtained less than $8 \mathrm{~min}$ downstream of quasi-perpendicular bow shocks satisfy a relation of the form Eq. (3) with values of $S$ and $\alpha$ that are not too different from those determined by Phan et al. (1994) in the vicinity of the magnetopause. This indicates that the state near marginal stability is already reached close to the shock.

Figure 5 shows that the largest amplitudes of the ioncyclotron waves at quasi-perpendicular low- $\beta$ shocks are observed on average about 5 min downstream of the keytime (see also Fig. 7). The bow shock moves relative to the spacecraft at speeds of $10-100 \mathrm{~km} \mathrm{~s}^{-1}$. Taking a typical speed of $30 \mathrm{~km} \mathrm{~s}^{-1}$, we can translate $5 \mathrm{~min}$ to a downstream distance of $9000 \mathrm{~km}$. For our events, the plasma velocity, $v_{p n}$, normal to quasi-perpendicular shock is $120 \mathrm{~km} \mathrm{~s}^{-1}$ on average. Thus, the plasma needs about $75 \mathrm{~s}$ to flow $9000 \mathrm{~km}$ downstream. Since the ion-cyclotron waves are convected with the plasma while they are growing, these $75 \mathrm{~s}$ can serve as a rough estimate for the time $\tau$ that the waves need to reach their maximum amplitudes and saturate. In terms of gyroperiods, we have $\tau \sim 75 \mathrm{~s} \approx 50 / f_{c p}$. Moreover, we find that on the same time scale $\tau$, the temperature anisotropy is reduced from about 2.5 immediately downstream of the low $\beta$ shock to about 2.1 (Fig. 4), and that the ion-cyclotron waves typically reach amplitudes of $\delta B / B \sim 0.07$.

These results can be compared with two-dimensional hybrid simulations of McKean et al. (1994). These authors examined a plasma with $\beta_{p \|}=1$ and $T_{p \perp} / T_{p \|}=3$. Under these conditions, the ion-cyclotron mode is found to be the dominant mode and the waves saturate after $\tau \approx 5 / f_{c p}$ and reach amplitudes of $\delta B / B \sim 0.15$. The proton temperature anisotropy is reduced on the same time scale $\tau$ from 3 to about 1.8. For our data set of low- $\beta$ bow shocks, $T_{p \perp} / T_{p \|}$ is, on average, 2.5 and $\beta_{p \|}$ is, on average, 0.5 , immediately downstream of the shock. Since these values are considerably lower than the initial values used by McKean et al. (1994), the plasma simulated by McKean et al. (1994) is initially farther away from the state of marginal stability. Thus, it is not surprising that the waves grow faster, reach larger amplitudes and therefore, lead to a stronger reduction of the anisotropy by means of pitch angle scattering.

McKean et al. (1994) also examined a plasma with $\beta_{p \|}=$ 4 and $T_{p \perp} / T_{p \|}=3$. Under these conditions, the ion-cyclotron mode dominates for low $\alpha$-particle concentration, whereas the mirror mode dominates for high $\alpha$-particle concentration. The waves saturate after $\tau \approx 10 / f_{c p}$ and reach amplitudes of $\delta B / B \sim 0.2$. The proton temperature anisotropy is reduced on the same time scale $\tau$ from 3 to about 1.5. This can be compared with data obtained at the quasi-perpendicular high $\beta$ shock. For our data set of high- $\beta$ bow shocks, $T_{p \perp} / T_{p \|}$ is, on average, 1.8 and $\beta_{p \|}$ is, on average, 5, immediately downstream of the shock. Again, the plasma simulated by McKean et al. (1994) is initially much farther away from the state of marginal stability. Figure 4 shows that a reduction of the anisotropy to 1.3 is observed $30 \mathrm{~s}$ downstream of keytime. This again can be translated to a downstream distance and used to estimate the time span that passes while the plasma travels this distance. This estimate gives $8 \mathrm{~s} \approx 2.5 / f_{c p}$. Finally, it should be noted that the mirror waves analyzed by Czaykowska et al. (1998) have amplitudes of $\delta B / B \sim 0.2$, which is comparable to those found in the simulations of McKean et al. (1994).

\section{Conclusions}

The nice new result of the present statistical study is the good correlation of the ratio of left-to-right hand polarized components of the magnetic wave activity and the anisotropy of the proton temperature. In addition, we conclude that the present study has, in general, confirmed earlier observations by Hubert et al. (1989, 1998), Lacombe et al. (1992, 1995), Fuselier et al. (1994), Schwartz et al. (1996) and others of low frequency magnetic wave modes in the magnetosheath adjacent to the bow shock. The basic two modes on which we have concentrated were the magnetic mirror and ion-cyclotron modes. In the statistical average, these modes show only weak signatures in the region of the magnetosheath investigated in this study. There are single occasions when the mirror mode is excited relatively strongly. 
Such cases have been reported by Lacombe et al. (1992) and have been verified by us in a more comprehensive case study (Czaykowska et al., 1998). Cases of moderately intense electromagnetic ion-cyclotron modes behind the quasiperpendicular bow shock had been reported already long ago by Sckopke et al. (1990). But in the average picture of the shock-adjacent magnetosheath, these cases for the two wave modes seem to be the rarer events, most likely with the electromagnetic ion-cyclotron waves being the more probable ones, whenever more energetic ions pass across the shock. This is not unreasonable as one does not expect very strong anisotropies in the protons to evolve here, as the plasma is not sufficiently compressed at the shock distance where one is located relatively far away from the obstacle. Generally, stronger compression is expected closer to the magnetopause where it is known that the mirror mode is developing more frequently and at larger amplitude. On the other hand, when transmitted energetic ions are present, as in Sckopke's et al. (1990) case, the anisotropy would increase and one may expect mirror modes to become unstable as well. When this is not the case, it is due to four effects. First, the transmitted ions are only a minor component in the plasma, which is probably not sufficient to raise the anisotropy high enough for exceeding the mirror threshold. Second, the anisotropy is, however, as Sckopke et al. (1990) have convincingly demonstrated, high enough for making the electromagnetic ioncyclotron wave unstable. This simply requires large enough resonant energy of the resonant particles, and a positive temperature anisotropy $\left[T_{p \perp} / T_{p \|}\right]_{\text {res }}-1>0$ for only the resonant particles, while the mirror mode requires, in addition, a bulk positive pressure anisotropy and satisfaction of the mirror criterion. The few resonant ions may not be in sufficient numbers to produce high enough bulk anisotropy. Third, the ion-cyclotron wave is known to compete heavily with the mirror mode and may, in many cases, win. This is the case during the fourth effect, when the plasma contains lots of warm or even hot electrons which Landau damp the mirror mode. This is certainly the case just behind the shock where electrons are heated very strongly. Further down, as they stream farther into the magnetosheath, the electrons cool due to several effects and close to the magnetopause, they are less dangerous for the mirror mode. The detailed explanation of the observations does, however, require more theoretical efforts. In particular, one general property of our spectra which we have not yet discussed, but which is remarkable in all of the sheath spectra, is that the spectra generally possess a break around $\sim 0.1 \mathrm{~Hz}$ with a relatively flat power law decay at lower than break frequency, and a steeper power law decay at higher than break frequency. This behaviour confirms that the magnetic turbulence in the magnetosheath, to some extent, is a scale-invariant feature. We will not discuss any interpretation of this well known fact at this point. There are several reasons for the generation of this kind of spectrum, one of which is the Doppler broadening of low frequency turbulence by high magnetosheath flow, in which case, the power spectra simply map the $k$-spectrum of the turbulence. The steep high-frequency decay has, in most cases, a power-law index between -2 and -3 , while the low frequency spectrum has a power-law index closer to -1 . In any case, interpretation of these laws is still missing.

Acknowledgements. G. Paschmann and H. Lühr were the principal investigators of the AMPTE/IRM plasma and magnetic field experiments, respectively. We appreciate valuable discussions with our late colleague N. Sckopke who passed away on 28 November 1999. We also thank the two referees for their helpful remarks.

Topical Editor thanks M. B. Bavassano Cattaneo and D. Hubert for their help in evaluating this paper.

\section{References}

Anderson, B. J. and Fuselier, S. A., Magnetic pulsations from 0.1 to 4.0 Hz and associated plasma properties in the Earth's subsolar magnetosheath and plasma depletion layer, J. Geophys. Res., 98, 1461-1479, 1993.

Anderson, B. J., Fuselier, S. A., Gary, S. P., and Denton, R. E., Magnetic spectral signatures in the Earth's magnetosheath and plasma depletion layer, J. Geophys. Res., 99, 5877-5891, 1994.

Bauer, T. M., Baumjohann, W., Treumann, R. A., Sckopke, N., and Lühr, H., Low-frequency waves in the near-Earth plasma sheet, J. Geophys. Res., 100, 9605-9617, 1995.

Bavassano-Cattaneo, M. B., Basile, C., Moreno, G., and Richardson, J. D., Evolution of mirror structures in the magnetosheath of Saturn from the bow shock to the magnetopause, J. Geophys. Res., 103, 11961-11972, 1998.

Belcher, J. W. and Davis, L., Large-amplitude Alfvén waves in the interplanetary medium, 2, J. Geophys. Res., 76, 3534-3563, 1971.

Blanco-Cano, X. and Schwartz, S. J., AMPTE-UKS observations of low frequency waves in the ion foreshock, Adv. Space Res., 15 (8/9), 97-101, 1995

Burgess, D., Cyclical behaviour of quasi-parallel collisionless shocks, Geophys. Res. Lett., 16, 345-349, 1989.

Czaykowska, A., Bauer, T. M., Treumann, R. A., and Baumjohann, W., Mirror waves downstream of the quasi-perpendicular bow shock, J. Geophys. Res., 103, 4747-4753, 1998.

Edmiston, J. P. and Kennel, C. F., A parametric survey of the first critical Mach number for a fast MHD shock, J. Plasma Phys., 32, 429-441, 1984.

Fuselier, S. A., Anderson, B. J., Gary, S. P., and Denton, R. E., Ion anisotropy/beta correlations in the Earth's quasi-parallel magnetosheath, J. Geophys. Res., 99, 14931-14936, 1994.

Gary, S. P., Low-frequency waves in a high-beta collisionless plasma: Polarization, compressibility and helicity, J. Plasma Phys., 35, 431-447, 1986.

Gary, S. P., Fuselier, S. A., and Anderson, B. J., Ion anisotropy instabilities in the magnetosheath, J. Geophys. Res., 98, 14811488, 1993.

Greenstadt, E. W. and Mellott, M. M., Plasma wave evidence for reflected ions in front of subcritical shocks: ISEE 1 and 2 observations, J. Geophys. Res., 92, 4730-4734, 1987.

Greenstadt, E. W., Le, G., and Strangeway, R. J., ULF waves in the foreshock, Adv. Space Res., 15 (8/9), 71-84, 1995.

Hasegawa, A., Drift mirror instability in the magnetosphere, Phys. Fluids, 12, 2642-2650, 1969.

Hasegawa, A., Plasma Instabilities and Nonlinear Effects, SpringerVerlag, New York, 1975. 
Hoppe, M. M., Russell, C. T., Frank, L. A., Eastman, T. E., and Greenstadt, E. W., Upstream hydromagnetic waves and their association with backstreaming ion populations: ISEE 1 and 2 observations, J. Geophys. Res., 86, 4471-4492, 1981.

Hubert, D., Perche, C., Harvey, C. C., Lacombe, C., and Russell, C. T., Observation of mirror waves downstream of a quasiperpendicular shock, Geophys. Res. Lett., 16, 159-162, 1989.

Hubert, D., Lacombe, C., Harvey, C. C., Moncuquet, M., Russell, C. T., and Thomsen, M. F., Nature, properties, and origin of low-frequency waves from an oblique shock to the inner magnetosheath, J. Geophys. Res., 103, 26783-26798, 1998.

Kennel, C. F., Edmiston, J. P., and Hada, T., A quarter century of collisionless shock research, in Collisionless Shocks in the Heliosphere: A Tutorial Review, Geophys. Monogr. Ser., 34, eds. R. G. Stone and B. T. Tsurutani, pp. 1-36, AGU, Washington, DC, 1985.

Kivelson, M. G. and Southwood, D. J., Mirror instability II: The mechanism of nonlinear saturation, J. Geophys. Res., 101, 17365-17374, 1996

Krauss-Varban, D., Waves associated with quasi-parallel shocks: Generation, mode conversion and implications, Adv. Space Res., 15 (8/9), 271-284, 1995.

Krauss-Varban, D. and Omidi, N., Structure of medium Mach number quasi-parallel shocks: Upstream and downstream waves, J. Geophys. Res., 96, 17715-17731, 1991.

Lacombe, C. F., Pantellini, G. E., Hubert, D., Harvey, C. C., Mangeney, A., Belmont, G., and Russell, C. T., Mirror and Alfvénic waves observed by ISEE 1-2 during crossings of the Earth's bow shock, Ann. Geophysicae, 10, 772-785, 1992.

Lacombe, C. F., Belmont, G., Hubert, D., Harvey, C. C., Mangeney, A., Russell, C. T., Gosling, J. T., and Fuselier, S. A., Density and magnetic field flucttuations observed by ISEE 1-2 in the quiet magnetosheath, Ann. Geophysicae, 13, 343-357, 1995.

Le, G. and Russell, C. T., A study of ULF wave foreshock morphology - II: Spatial variation of ULF waves, Planet. Space Sci., 40, 1215-1225, 1992.

Lee, L. C., Price, C. P., Wu, C. S., and Mandt, M. E., A study of mirror waves generated downstream of a quasi-perpendicular shock, J. Geophys. Res., 93, 247-250, 1988.

Lühr, H., Klöcker, N., Oelschlägel, W., Häusler, B., and Acuña, M., The IRM fluxgate magnetometer, IEEE Trans. Geosci. Remote Sens., GE-23, 259-261, 1985.

McKean, M. E., Winske, D., and Gary, S. P., Two-dimensional simulations of ion anisotropy instabilities in the magnetosheath, J. Geophys. Res., 99, 11141-11153, 1994.

McKenzie, J. F. and Westphal, K. O., Transmission of Alfvén waves through the Earth's bow shock, Planet. Space Sci., 17, 1029$1037,1969$.

Mellott, M. M. and Livesey, W. A., Shock overshoots revisited, J. Geophys. Res., 92, 13661-13665, 1987.

Omidi, N., How the bow shock does it, Rev. Geophys., Supplement, 629-637, 1995.

Pantellini, F. G. E., A model of the formation of stable nonpropa- gating magnetic structures in the solar wind based on the nonlinear mirror instability, J. Geophys. Res., 103, 4789-4798, 1998.

Pantellini, F. G. E. and Schwartz, S. J., Electron temperature effects in the linear proton mirror instability, J. Geophys. Res., 100, 3539-3545, 1995

Paschmann, G., Loidl, H., Obermayer, P., Ertl, M., Laborenz, R., Sckopke, N., Baumjohann, W., Carlson, C. W., and Curtis, D. W. The plasma instrument for AMPTE/IRM, IEEE Trans. Geosci. Remote Sens., GE-23, 262-266, 1985.

Phan, T.-D., Paschmann, G., Baumjohann, W., Sckopke, N., and Lühr, H., The magnetosheath region adjacent to the dayside magnetopause: AMPTE/IRM observations, J. Geophys. Res., 99, 121-141, 1994.

Pokhotelov, O. A., Balikhin, M. A., Alleyne, H. St.-C., and Onishchenko, O. G., Mirror instability with finite electron temperature effects, J. Geophys. Res., 105, 2393-2402, 2000a.

Pokhotelov, O. A., Balikhin, M. A., Treumann, R. A., and Onishchenko, O. G., Kinetic theory of the mirror instability including electron temperature effects, J. Geophys. Res., accepted, 2000b.

Price, C. P., Swift, D. W., and Lee, L.-C., Numerical simulations of nonoscillatory mirror waves at the Earth's magnetosheath, J. Geophys. Res., 91, 101-112, 1986.

Russell, C. T. and Farris, M. H., Ultra low frequency waves at the Earth's bow shock, Adv. Space Res., 15 (8/9), 285-296, 1995.

Scholer, M., Kucharek, H., and Jayanti, V., Waves and turbulence in high Mach number nearly parallel collisionless shocks, J. Geophys. Res., 102, 9821-9833, 1997.

Schwartz, S. J., Burgess, D., and Moses, J. J., Low-frequency waves in the Earth's magnetosheath: Present status, Ann. Geophysicae, 14, 1134-1150, 1996.

Sckopke, N., Paschmann, G., Bame, S. J., Gosling, J. T., and Russell, C. T., Evolution of ion distributions across the nearly perpendicular bow shock: Specularly and nonspecularly reflected ions, J. Geophys. Res., 88, 6121-6136, 1983.

Sckopke, N., Paschmann, G., Brinca, A. L., Carlson, C. W., and Lühr, H., Ion thermalization in quasi-perpendicular shocks involving reflected ions, J. Geophys. Res., 95, 6337-6352, 1990.

Stone, R. G. and Tsurutani, B. T. (eds.), Collisionless shocks in the Heliosphere: A tutorial review, Geophys. Monogr. 34, AGU, Washington DC, 1985.

Thomsen, M. F., Gosling, J. T., and Bame, S. J., Ion and electron heating at collisionless shocks near the critical Mach number, J. Geophys. Res., 90, 137-148, 1985.

Treumann, R. A. and Baumjohann, W., Advanced Space Plasma Physics, pp. 211-217, Imperial College Press, London, 1997.

Tsurutani, B. T. and Stone, R. G. (eds.), Collisionless shocks in the heliosphere: Review of current research, Geophys. Monogr., 35, AGU, Washington DC, 1985.

Winske, D., Omidi, N., Quest, K. B., and Thomas, V. A., Reforming supercritical quasi-parallel shocks, 2, Mechanism for wave generation and front re-formation, J. Geophys. Res., 95, 18821-18832, 1990. 